\title{
Is Peak Concentration Needed in Therapeutic Drug Monitoring of Vancomycin? A Pharmacokinetic-Pharmacodynamic Analysis in Patients with Methicillin-Resistant Staphylococcus aureus Pneumonia
}

\author{
Yosuke Suzuki $^{\mathrm{a}} \quad$ Kanako Kawasaki $^{\mathrm{a}}$ Yuhki Sato $^{\mathrm{a}}$ Issei Tokimatsu ${ }^{\mathrm{b}}$ Hiroki Itoh $^{\mathrm{a}}$ \\ Kazufumi Hiramatsu ${ }^{b}$ Masaharu Takeyama ${ }^{a}$ Jun-ichi Kadotab \\ a Department of Clinical Pharmacy, Oita University Hospital, and ${ }^{b}$ Internal Medicine II, Oita University Faculty of Medicine, \\ Oita, Japan
}

\section{Key Words}

Vancomycin - Therapeutic drug monitoring • Peak concentration - Pharmacokinetic-pharmacodynamic relationship

\begin{abstract}
Background: We analyzed the pharmacokinetic-pharmacodynamic relationship of vancomycin to determine the drug exposure parameters that correlate with the efficacy and nephrotoxicity of vancomycin in patients with methicillinresistant Staphylococcus aureus pneumonia and evaluated the need to use peak concentration in therapeutic drug monitoring (TDM). Methods: Serum drug concentrations of 31 hospitalized patients treated with vancomycin for methicillin-resistant S. aureus pneumonia were collected. Results: Significant differences in trough concentration $\left(C_{\min }\right) /$ minimum inhibitory concentration (MIC) and area under the serum concentration-time curve $\left(\mathrm{AUC}_{0-24}\right) / \mathrm{MIC}$ were observed between the response and non-response groups. Significant differences in $\mathrm{C}_{\min }$ and $\mathrm{AUC}_{0-24}$ were observed between the nephrotoxicity and non-nephrotoxicity groups. Receiver operating characteristic curves revealed high predictive values of $C_{\min } / \mathrm{MIC}$ and $\mathrm{AUC}_{0-24} / \mathrm{MIC}$ for efficacy and of $C_{\min }$ and $\mathrm{AUC}_{0-24}$ for safety of vancomycin. Conclusions: These results
\end{abstract}

suggest little need to use peak concentration in vancomycin TDM because $C_{\min } / \mathrm{MIC}$ and $C_{\min }$ are sufficient to predict the efficacy and safety of vancomycin.

Copyright ๑ 2012 S. Karger AG, Basel

\section{Introduction}

Throughout the world, the treatment of infectious diseases caused by Gram-positive bacteria has been complicated by the emergence of bacteria resistant to $\beta$-lactam, macrolide and fluoroquinolone antibacterial drugs [1-3]. Vancomycin is a glycopeptide antibiotic and one of the most widely used antibiotics for the treatment of serious infections by methicillin-resistant Staphylococcus aureus (MRSA) [4]. Recent vancomycin therapeutic monitoring guidelines recommend more aggressive vancomycin dosing regimens and maintaining vancomycin trough concentrations $\left(\mathrm{C}_{\min }\right)$ between 15 and $20 \mu \mathrm{g} / \mathrm{ml}$, aiming to achieve optimal target serum vancomycin concentrations and improve clinical outcomes [5]. Given the link with pharmacodynamics, several studies have revealed that the area under the serum concentration-time curve (AUC)/minimum inhibitory concentration (MIC) is the preferred parameter for therapeutic monitoring based in

\section{KARGER}

Fax +41 613061234 E-Mail karger@karger.ch www.karger.com (c) 2012 S. Karger AG, Basel

0009-3157/12/0584-0308\$38.00/0

Accessible online at:

www.karger.com/che
Yosuke Suzuki

Department of Clinical Pharmacy

Oita University Hospital

Hasama-machi, Oita 879-5593 (Japan)

E-Mail y-suzuki@oita-u.ac.jp 
part on data obtained from animal models, in vitro studies and limited human studies [6-8]. On the other hand, a recent prospective multicenter trial has suggested that vancomycin $C_{\min }>15 \mu \mathrm{g} / \mathrm{ml}$ at steady state is a risk factor for nephrotoxicity [9]. Thus, $\mathrm{C}_{\min }$ or AUC may be useful for therapeutic drug monitoring (TDM) of vancomycin.

Blood samplings at several time points are necessary for the accurate prediction of AUC. However, serial serum vancomycin concentrations are difficult to obtain in the clinical setting. In Japan, peak concentration $\left(\mathrm{C}_{\max }\right)$ and $\mathrm{C}_{\min }$ of vancomycin are measured routinely for vancomycin TDM [10]. $\mathrm{C}_{\max }$ is useful to predict the AUC, but it may be unnecessary if $\mathrm{C}_{\min }$ and AUC are equally useful for predicting vancomycin efficacy. In this study, we analyzed the pharmacokinetic-pharmacodynamic relationship of vancomycin to determine the drug exposure parameters that correlate with the efficacy and safety of vancomycin and to evaluate whether $\mathrm{C}_{\max }$ is needed for TDM in patients with MRSA pneumonia.

\section{Patients and Methods}

\section{Patients}

Medical records were reviewed to identify hospitalized patients treated with vancomycin for MRSA pneumonia at Oita University Hospital between June 2005 and December 2011. Patients who were younger than 18 years of age, hemodialyzed or received other anti-MRSA agents were excluded. MRSA pneumonia was diagnosed based on the following criteria: radiographically documented hospital-acquired pneumonia, sputum specimen positive for MRSA, fever and elevated white blood cell count or C-reactive protein. Serum drug concentrations (trough and peak) were obtained from routine TDM data. An average of 2.8 (range 2-6) serum vancomycin level data were available from each patient. The following clinical data recorded on the first day of vancomycin administration and on the same day as TDM were also collected: gender, age, body weight and serum creatinine. Creatinine clearance was calculated according to the Cockcroft-Gault equation [11]. This study was approved by the ethics committee of Oita University Hospital. Since blood samples were collected as part of the routine patient care for TDM and laboratory testing, written informed consent was not necessary.

\section{Drug Concentration Monitoring}

Vancomycin was infused intravenously over $1-2 \mathrm{~h}$. Venous blood samples were collected within an hour before the next administration and from 1 to $2 \mathrm{~h}$ after vancomycin infusion. The exact times of dosing and blood sampling were always recorded. Vancomycin assay was performed as part of the routine laboratory test at Oita University Hospital. Serum vancomycin concentrations were determined by a particle-enhanced turbidimetric inhibition immunoassay based on Dimension ${ }^{\circledR}$ Xpand (Siemens Inc., Ill., USA). With this method, the limit of detection was 0.8 $\mu \mathrm{g} / \mathrm{ml}$ and the coefficient of variation was $<5 \%$ for routine clinical TDM. Furthermore, the measurement error was $<10 \%$ in se- rum samples containing $30 \mathrm{mg} / \mathrm{dl}$ creatinine and $500 \mathrm{mg} / \mathrm{dl}$ urea, indicating that the measurements were not affected by the renal function of the patients.

\section{Estimation of Pharmacokinetic Parameters}

Pharmacokinetics of vancomycin were analyzed using a twocompartment model, with elimination of the central compartment. The Bayesian forecasting method was employed to estimate individual pharmacokinetic parameters including total body clearance, volume of distribution at steady state, transfer rate constant from central to peripheral, and transfer rate constant from peripheral to central, using individual serum drug concentrations and the population parameters [10]. The estimated parameters allowed prediction of an individual serum concentration-time curve and estimation of AUC from time 0 to $24 \mathrm{~h}\left(\mathrm{AUC}_{0-24}\right), \mathrm{C}_{\max }$ and $C_{\min }$. The $C_{\max }$ and $C_{\min }$ values were estimated for individual patients at the end of infusion and immediately before starting the next infusion, respectively. The highest $\mathrm{C}_{\max }, \mathrm{C}_{\min }$ and $\mathrm{AUC}_{0-24}$ values during the treatment period were used to analyze the potential association with efficacy and nephrotoxicity of vancomycin, because these individual values varied during treatment due to changes in dose and dosing interval according to TDM.

Determination of Bacteriological Efficacy and MIC

MRSA strains were identified by the Clinical Microbiology Laboratory in Oita University Hospital. The viable counts (colony-forming units) of MRSA in patient's sputum samples were quantitatively determined before and $>4$ days after the first vancomycin infusion. Bacteriological efficacy was classified as follows: (1) excellent response was defined as eradication of MRSA; (2) good response was defined as a reduction in viable count of $>1$ $\log _{10}$ colony-forming units, and (3) poor response was defined as $<1 \log _{10}$ reduction, no change or increase in viable count. Patients with excellent and good responses were classified as the response group, and those with poor response as the non-response group. The MIC of vancomycin was determined by the broth microdilution method according to the guidelines of the National Committee for Clinical Laboratory Standards [12].

\section{Evaluation of Nephrotoxicity}

Occurrence of nephrotoxicity was defined as an increase in serum creatinine level of $0.5 \mathrm{mg} / \mathrm{dl}$ or a $50 \%$ increase, whichever was greater, on at least 2 consecutive days during the period from initiation of vancomycin therapy to $72 \mathrm{~h}$ after the completion of therapy [13].

Pharmacokinetic-Pharmacodynamic Analysis and Statistics

For the analysis of bacteriological efficacy, pharmacokineticpharmacodynamic parameters including $\mathrm{C}_{\max } / \mathrm{MIC}, \mathrm{C}_{\min } / \mathrm{MIC}$ and $\mathrm{AUC}_{0-24} / \mathrm{MIC}$ were compared between response and non-response groups. Both MIC and pharmacokinetic-pharmacodynamic indices were assumed to show a log normal distribution. Therefore, the values for these variables were transformed to natural logarithm. For the analysis of nephrotoxicity, pharmacokinetic parameters including $\mathrm{C}_{\max }, \mathrm{C}_{\min }$ and $\mathrm{AUC}_{0-24}$ were compared between the presence (nephrotoxicity group) and absence of nephrotoxicity (non-nephrotoxicity group). Differences between the two groups were analyzed by two-sided Student's t test. The relationship between observed and predicted concentrations in individuals was analyzed by Pearson's product-moment cor- 
Table 1. Patient characteristics at the first day of vancomycin administration and their drug exposure parameters

\begin{tabular}{lc}
\hline Characteristic & Value \\
\hline Subjects & 31 \\
Males/females & $28 / 3$ \\
Age, years & $73.0 \pm 9.2(54-87)$ \\
Body weight, kg & $52.8 \pm 11.4(35.7-77.0)$ \\
Blood urea nitrogen, mg/dl & $23.4 \pm 6.5(13.4-34.9)$ \\
Serum creatinine, mg/dl & $1.2 \pm 0.4(0.67-2.06)$ \\
Creatinine clearance, $\mathrm{ml} / \mathrm{min}$ & $61.8 \pm 31.4(24.0-153.5)$ \\
Serum vancomycin & \\
$\quad$ level data per patient, $\mathrm{n}$ & $2.8 \pm 1.1(2-6)$ \\
$\mathrm{MIC} \mu \mathrm{g} / \mathrm{ml}$ & $0.92 \pm 0.19(0.5-1)$ \\
$\mathrm{C}_{\max }, \mu \mathrm{g} / \mathrm{ml}$ & $42.5 \pm 9.9(19.3-62.2)$ \\
$\mathrm{C}_{\min }, \mu \mathrm{g} / \mathrm{ml}$ & $16.3 \pm 6.8(3.2-29.8)$ \\
$\mathrm{AUC}_{0-24}, \mu \mathrm{g} \cdot \mathrm{h} / \mathrm{ml}$ & $540.6 \pm 165.0(214.6-890.3)$ \\
$\mathrm{C}_{\max } / \mathrm{MIC}$ & $49.1 \pm 18.0(19.3-98.3)$ \\
$\mathrm{C}_{\min } / \mathrm{MIC}$ & $19.0 \pm 10.0(3.2-45.8)$ \\
$\mathrm{AUC}_{0-24} / \mathrm{MIC}$ & $629.1 \pm 272.8(214.6-1,320.6)$ \\
\end{tabular}

Data are expressed as the mean $\pm \mathrm{SD}$, with ranges in parentheses. Creatinine clearance was calculated according to the Cockcroft-Gault equation.

relation coefficient. To evaluate the diagnostic accuracy of the pharmacokinetic-pharmacodynamic parameters, receiver operating characteristic (ROC) curve and area under the ROC curve $\left(\right.$ AUC $\left._{\mathrm{ROC}}\right)$ were analyzed. All values are expressed as the mean \pm SD. A p value $<0.05$ was considered statistically significant. Statistical analyses were performed using the $\mathrm{R}$ software version 2.14.2 (http://www.r-project.org) and Predictive Analysis Software Statistics version 18 (SPSS Inc., Chicago Ill., USA).

\section{Results}

A review of patient records identified 31 patients who satisfied the inclusion criteria. Table 1 describes the characteristics of these patients on the first day of vancomycin administration. Of the 31 patients, 23 were assessed as showing response (response group) and 8 as showing no response (non-response group). The MIC of vancomycin was $0.5 \mu \mathrm{g} / \mathrm{ml}$ in 5 patients and $1 \mu \mathrm{g} / \mathrm{ml}$ in 18 patients in the response group, while the MIC was $1 \mu \mathrm{g} / \mathrm{ml}$ in all patients in the non-response group. The duration of vancomycin treatment was $12.3 \pm 4.4$ and $11.4 \pm 4.2$ days in the response and non-response group, respectively, with no significant difference between the two groups ( $\mathrm{p}=$ $0.40)$. On the other hand, nephrotoxicity was observed in 7 patients. The duration of vancomycin treatment was $12.9 \pm 5.2$ and $11.9 \pm 4.1$ days in patients with nephro- toxicity and without nephrotoxicity, respectively, and did not differ significantly between the two groups $(p=0.60)$. Nephrotoxicity occurred $9.7 \pm 4.8$ days after initiation of vancomycin, after the period of blood sampling for TDM (5.0 \pm 2.9 days). Scatter plots showed a good correlation between the observed and predicted concentrations of individuals $(y=0.96 x+0.48 ; r=0.95)$.

Figure 1 shows the box and whisker plots of the pharmacokinetic-pharmacodynamic parameters in the response and non-response groups (fig. $1 \mathrm{a}-\mathrm{c}$ ) and the pharmacokinetic parameters in the nephrotoxicity and nonnephrotoxicity groups (fig. 1d-f). Significant differences in $\mathrm{C}_{\mathrm{min}} / \mathrm{MIC}$ and $\mathrm{AUC}_{0-24} / \mathrm{MIC}$ ( $\mathrm{p}=0.049$ and 0.044 , respectively) were observed between the response and nonresponse group, while no significant difference in $\mathrm{C}_{\max } /$ MIC ( $p=0.222$ ) was detected between the two groups. Significant differences in $\mathrm{C}_{\min }$ and $\mathrm{AUC}_{0-24}(\mathrm{p}=0.011$ and 0.014 , respectively) were observed between the nephrotoxicity and non-nephrotoxicity group, while no significant difference in $\mathrm{C}_{\max }(\mathrm{p}=0.498)$ was detected between the two groups. Figure 2 a shows the ROC curves for the efficacy of vancomycin comparing the pharmacokineticpharmacodynamic parameters. The $\mathrm{AUC}_{\mathrm{ROC}}$ was 0.67, 0.77 and 0.76 for $\mathrm{C}_{\max } / \mathrm{MIC}, \mathrm{C}_{\min } / \mathrm{MIC}$ and $\mathrm{AUC}_{0-24} / \mathrm{MIC}$, respectively. Similarly, figure $2 \mathrm{~b}$ shows the ROC curves for nephrotoxicity associated with vancomycin comparing the pharmacokinetic parameters. The $\mathrm{AUC}_{\mathrm{ROC}}$ was 0.63, 0.84 and 0.83 for $\mathrm{C}_{\max }, \mathrm{C}_{\min }$ and $\mathrm{AUC}_{0-24}$, respectively.

\section{Discussion}

Conventionally, $\mathrm{C}_{\max }$ together with $\mathrm{C}_{\min }$ are considered to be necessary in vancomycin TDM [5]. Geraci [14] recommended a $C_{\max }$ range of $30-40 \mu \mathrm{g} / \mathrm{ml}$, but did not provide the basis of how it was derived. Recent vancomycin therapeutic monitoring guidelines recommend to measure $C_{\min }$ and to maintain $C_{\min }$ between 15 and 20 $\mu \mathrm{g} / \mathrm{ml}$. From the pharmacokinetic-pharmacodynamic viewpoint, several studies have proposed AUC/MIC as the preferred parameter [6-8]. An $\mathrm{AUC}_{0-24} / \mathrm{MIC} \geq 400$ was recommended to optimize the pharmacokineticpharmacodynamic properties of vancomycin $[15,16]$. Estimation of $\mathrm{AUC}$ is incomplete without the $\mathrm{C}_{\max }$; thus, blood samplings at 2 or more time points (including trough and peak) are necessary to evaluate AUC/MIC as an index for vancomycin efficacy. In this study, we attempted to evaluate the need to use $\mathrm{C}_{\max }$ in vancomycin TDM by analyzing the pharmacokinetic-pharmacodynamic relationship with efficacy and safety. 


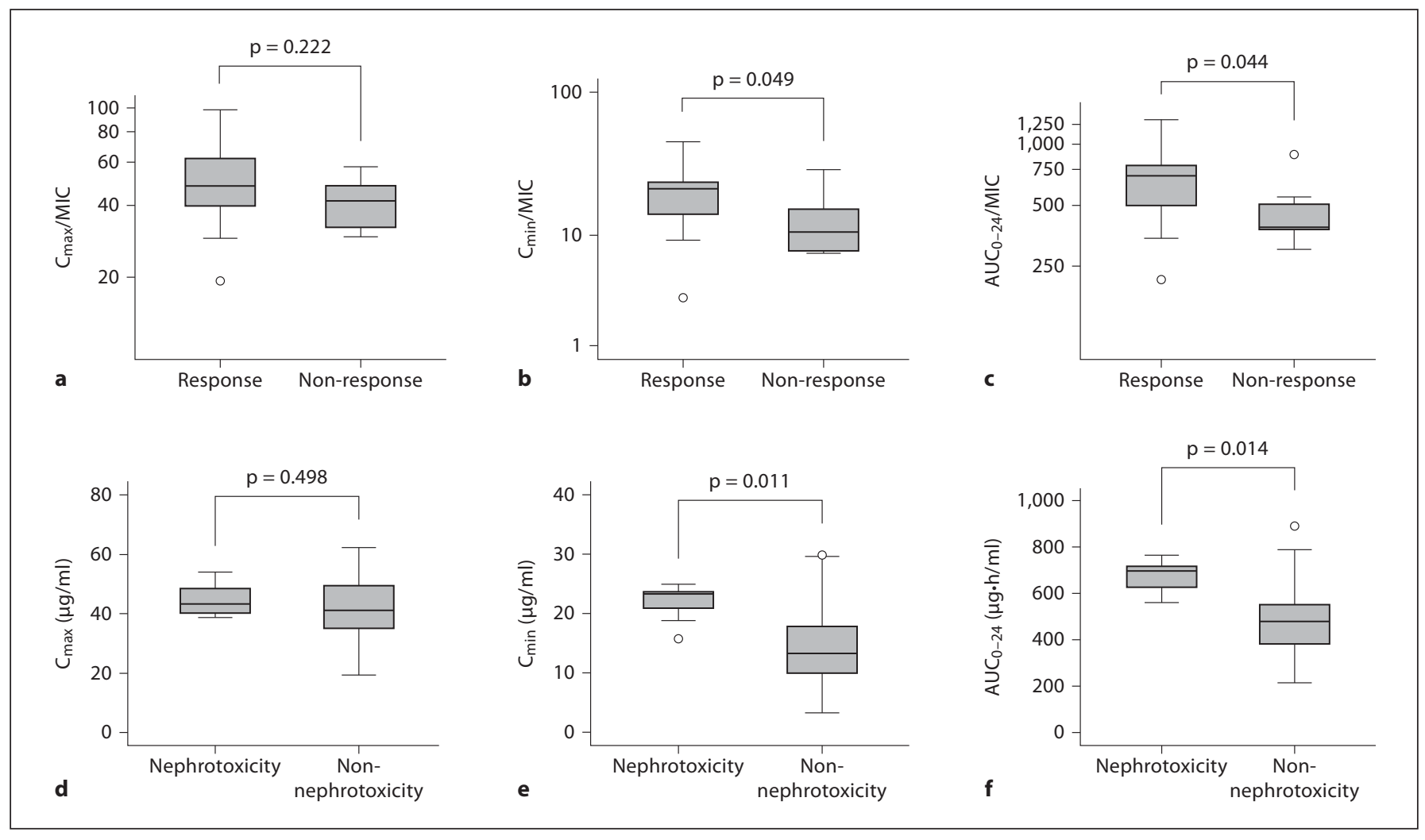

Fig. 1. Box and whisker plots of the pharmacokinetic-pharmacodynamic parameters in response and non-response groups (a-c) and pharmacokinetic parameters in nephrotoxicity and non-nephrotoxicity groups (d-f). Boxes indicate 25th, 50th and 75 th percentiles, and whiskers show \pm 1.5 times the interquartile range. $\bigcirc=$ Outlier.

Fig. 2. ROC curves for the efficacy of vancomycin comparing various pharmacokinetic-pharmacodynamic parameters (a) and for nephrotoxicity associated with vancomycin comparing different pharmacokinetic parameters (b).

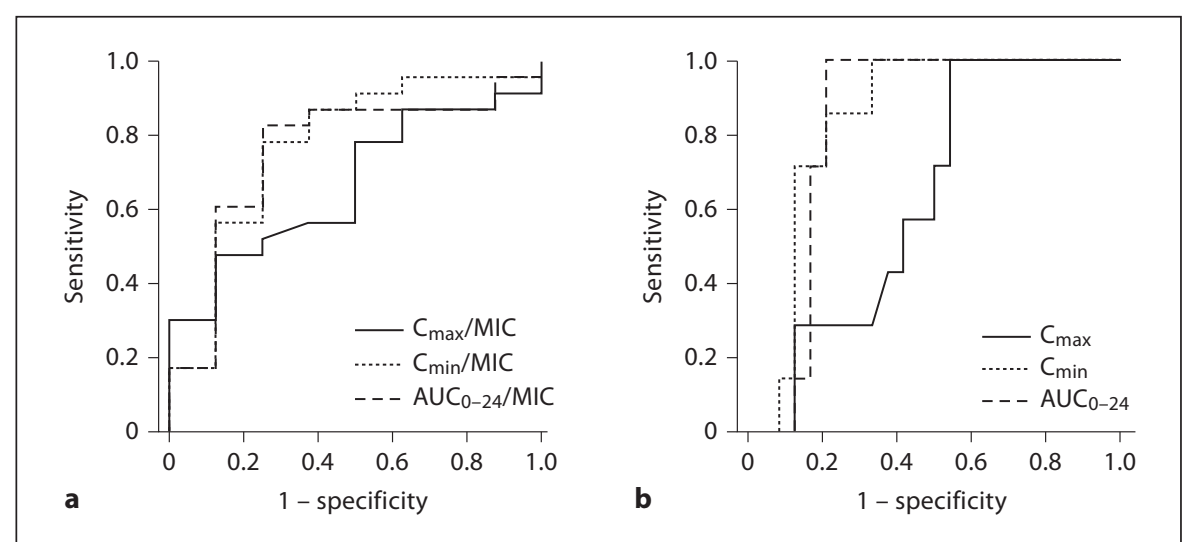

This study enrolled only patients with MRSA pneumonia not receiving any other anti-MRSA agents. This selection criterion precludes the bias due to differences in pathological conditions and other coadministered antiMRSA agents. We selected bacteriological response as the criterion for efficacy of vancomycin, which allows more precise evaluation of the efficacy of vancomycin against MRSA pneumonia. To evaluate the adverse effect of vancomycin, we selected nephrotoxicity in view of the high incidence and clinical importance.

Vancomycin therapy was effective in approximately $74.2 \%$ of the patients in this study. Significant differences 
in $\mathrm{C}_{\min } / \mathrm{MIC}$ and $\mathrm{AUC}_{0-24} / \mathrm{MIC}$ were observed between the response and non-response group, while no significant difference in $\mathrm{C}_{\max } / \mathrm{MIC}$ was found between the two groups. In addition, ROC curves revealed equally high predictive powers for both $\mathrm{C}_{\min } / \mathrm{MIC}$ and $\mathrm{AUC}_{0-24} / \mathrm{MIC}$, which were superior to that of $\mathrm{C}_{\max } / \mathrm{MIC}$. These results indicate that $\mathrm{C}_{\text {min }} / \mathrm{MIC}$ and $\mathrm{AUC}_{0-24} / \mathrm{MIC}$ are equally useful in predicting the efficacy of vancomycin in patients with MRSA pneumonia. Incidentally, no significant differences in both $\mathrm{C}_{\text {min }}$ and $\mathrm{AUC}_{0-24}$ were observed between the response and non-response group $(\mathrm{p}=0.186$ and 0.212 , respectively). This finding suggests that pharmacokinetic parameters alone are not useful to predict response, but pharmacokinetic-pharmacodynamic parameters $\left(\mathrm{C}_{\min } / \mathrm{MIC}\right.$ and $\left.\mathrm{AUC}_{0-24} / \mathrm{MIC}\right)$ that take into account both pharmacokinetics of vancomycin and sensitivity of the MRSA strain infecting the patient are necessary to predict the efficacy of vancomycin treatment for MRSA infection in each individual. On the other hand, nephrotoxicity was observed in approximately $22.6 \%$ of the patients in this study. A review demonstrated that nephrotoxicity associated with vancomycin therapy was observed in $2-28 \%$ of patients [17]. The dose of vancomycin was relatively high in this study because all patients had MRSA pneumonia, which may account for the relatively high incidence of nephrotoxicity. Significant differ- ences in $\mathrm{C}_{\min }$ and $\mathrm{AUC}_{0-24}$ were observed between the nephrotoxicity and non-nephrotoxicity group. In addition, $\mathrm{ROC}$ curves revealed equally high predictive powers for both $\mathrm{C}_{\min }$ and $\mathrm{AUC}_{0-24}$, which were superior to that of $\mathrm{C}_{\max }$. These results indicate that $\mathrm{C}_{\min }$ and $\mathrm{AUC}_{0-24}$ are equally useful in predicting the safety of vancomycin in patients with MRSA pneumonia. Therefore, our results do not support the necessity to use $\mathrm{C}_{\max }$ for vancomycin TDM because $\mathrm{C}_{\min } / \mathrm{MIC}$ and $\mathrm{C}_{\min }$ are sufficient to predict the efficacy and safety of vancomycin. This is the first report indicating that $\mathrm{C}_{\max }$ has little use in vancomycin TDM, and our finding may contribute to reduce the labor and cost in vancomycin TDM. However, the present study has some limitations: the study was retrospective and analyzed a small number of patients. Further, a largescale prospective study is required to verify whether a change in practice to measure trough level and MIC is appropriate in vancomycin TDM.

In conclusion, we evaluated the need to use $\mathrm{C}_{\max }$ for TDM of vancomycin by analyzing the pharmacokineticpharmacodynamic relationship with efficacy and safety. In this study, $\mathrm{C}_{\mathrm{min}} / \mathrm{MIC}$ and $\mathrm{AUC}_{0-24} / \mathrm{MIC}$ were equally powerful to predict efficacy while $\mathrm{C}_{\min }$ and $\mathrm{AUC}_{0-24}$ were equally powerful to predict safety of vancomycin in patients with MRSA pneumonia. These results suggest that there is little need to use $\mathrm{C}_{\max }$ in vancomycin TDM.

\section{References}

1 Rivera AM, Boucher HW: Current concepts in antimicrobial therapy against select Gram-positive organisms: methicillin-resistant Staphylococcus aureus, penicillin-resistant pneumococci, and vancomycin-resistant enterococci. Mayo Clin Proc 2011;86: 1230-1243.

-2 Sanfilippo CM, Hesje CK, Haas W, et al: Topoisomerase mutations that are associated with high-level resistance to earlier fluoroquinolones in Staphylococcus aureus have less effect on the antibacterial activity of besifloxacin. Chemotherapy 2011;57:363-371.

$\checkmark 3$ Yoon EJ, Lee CY, Shim MJ, et al: Extended spectrum of quinolone resistance, even to a potential latter third-generation agent, as a result of a minimum of two GrlA and two GyrA alterations in quinolone-resistant Staphylococcus aureus. Chemotherapy 2010; 56:153-157.

44 Moellering RC Jr: Vancomycin: a 50-year reassessment. Clin Infect Dis 2006;42(suppl 1):S3-S4.

5 Rybak M, Lomaestro B, Rotschafer JC, et al: Therapeutic monitoring of vancomycin in adult patients: a consensus review of the American Society of Health-System Phar- macists, the Infectious Diseases Society of America, and the Society of Infectious Diseases Pharmacists. Am J Health Syst Pharm 2009;66:82-98.

6 Rybak MJ: The pharmacokinetic and pharmacodynamic properties of vancomycin. Clin Infect Dis 2006;42(suppl 1):S35-S39.

7 Craig WA: Basic pharmacodynamics of antibacterials with clinical applications to the use of beta-lactams, glycopeptides, and linezolid. Infect Dis Clin North Am 2003;17:479-501.

$\checkmark 8$ Craig WA: Pharmacokinetic/pharmacodynamic parameters: rationale for antibacterial dosing of mice and men. Clin Infect Dis 1998;26:1-10.

9 Bosso JA, Nappi J, Rudisill C, et al: Relationship between vancomycin trough concentrations and nephrotoxicity: a prospective multicenter trial. Antimicrob Agents Chemother 2011;55:5475-5479.

10 Yasuhara M, Iga T, Zenda H, et al: Population pharmacokinetics of vancomycin in Japanese adult patients. Ther Drug Monit 1998; 20:139-148.

11 Cockcroft DW, Gault MH: Prediction of creatinine clearance from serum creatinine. Nephron 1976;16:31-41.
12 Methods for Dilution Antimicrobial Susceptibility Test for Bacteria that Grow Aerobically. Approved Standard M7-A5, ed 5. Wayne, National Committee for Clinical Laboratory Standards, 2000.

13 Lodise TP, Lomaestro B, Graves J, et al: Larger vancomycin doses (at least four grams per day) are associated with an increased incidence of nephrotoxicity. Antimicrob Agents Chemother 2008;52:1330-1336

14 Geraci JE: Vancomycin. Mayo Clin Proc 1977;52:631-634.

15 Moise-Broder PA, Forrest A, Birmingham MC, et al: Pharmacodynamics of vancomycin and other antimicrobials in patients with Staphylococcus aureus lower respiratory tract infections. Clin Pharmacokinet 2004;43:925-942.

16 Kullar R, Davis SL, Levine DP, et al: Impact of vancomycin exposure on outcomes in patients with methicillin-resistant Staphylococcus aureus bacteremia: support for consensus guidelines suggested targets. Clin Infect Dis 2011;52:975-981.

$>17$ Wong-Beringer A, Joo J, Tse E, et al: Vancomycin-associated nephrotoxicity: a critical appraisal of risk with high-dose therapy. Int J Antimicrob Agents 2011;37:95-101. 